\title{
Safety, pharmacokinetics and efficacy findings in an open-label, single-arm study of weekly paclitaxel plus lapatinib as first-line therapy for Japanese women with HER2-positive metastatic breast cancer
}

\author{
Kenichi Inoue ${ }^{1} \cdot$ Katsumasa Kuroi $^{2} \cdot$ Satoru Shimizu $^{3} \cdot$ Yoshiaki Rai $^{4} \cdot$ Kenjiro Aogi $^{5}$ • \\ Norikazu Masuda $^{6}$. Takahiro Nakayama ${ }^{7,8} \cdot$ Hiroji Iwata ${ }^{9}$ Yuichiro Nishimura ${ }^{10}$. \\ Alison Armour ${ }^{11} \cdot$ Yasutsuna Sasaki $^{12,13}$
}

Received: 23 January 2015 / Accepted: 14 April 2015 / Published online: 13 May 2015

(C) The Author(s) 2015. This article is published with open access at Springerlink.com

\begin{abstract}
Background Lapatinib is the human epidermal growth factor receptor 2 (HER2) targeting agent approved globally for HER2-positive metastatic breast cancer (MBC). The efficacy, safety and pharmacokinetics (PK) of lapatinib combined with paclitaxel $(\mathrm{L}+\mathrm{P})$ were investigated in this study, to establish clear evidence regarding the combination in Japanese patients. Methods In this two-part, single-arm, open-label study, the tolerability of $\mathrm{L}+\mathrm{P}$ as first-line treatment in Japanese patients with HER2-positive MBC was evaluated in six patients in the first part, and the safety, efficacy and PK were evaluated in a further six patients (making a total of twelve patients) in the second part. Eligible women were enrolled and received lapatinib $1500 \mathrm{mg}$ once daily and paclitaxel $80 \mathrm{mg} / \mathrm{m}^{2}$ weekly for at least 6 cycles.

Results The only dose-limiting toxicity reported was Grade 3 diarrhea in one patient. The systemic exposure to
\end{abstract}

Kenichi Inoue

ino@cancer-c.pref.saitama.jp

1 Division of Breast Oncology, Saitama Cancer Center, 780 Komuro, Ina-machi, Kita-adachi-gun, Saitama 362-0806, Japan

2 Department of Breast Surgery, Tokyo Metropolitan Cancer and Infectious Diseases Center Komagome Hospital, Tokyo, Japan

3 Department of Breast Oncology and Endocrine Surgery, Kanagawa Cancer Center, Yokohama, Kanagawa, Japan

4 Hakuaikai Medical Corporation, Sagara Hospital, Kagoshima, Japan

5 Department of Surgery, National Hospital Organization Shikoku Cancer Center, Matsuyama, Japan

6 Department of Surgery, Breast Oncology, National Hospital Organization Osaka National Hospital, Osaka, Japan maximum plasma concentration and area under the plasma concentration curve (AUC) for lapatinib, as well as the AUC of paclitaxel, were increased when combined. The most common adverse events (AEs) related to the study treatment were alopecia, diarrhea and decreased hemoglobin. The majority of drug-related AEs were Grade 1 or 2 . The median overall survival was 35.6 months (95\% confidence interval 23.9, not reached). The response rate and clinical benefit rate were both $83 \%$ (95\% confidence interval 51.6, 97.9).

Conclusions The $\mathrm{L}+\mathrm{P}$ treatment was well tolerated in Japanese patients with HER2-positive MBC. Although the PK profiles of lapatinib and paclitaxel influenced each other, the magnitudes were not greatly different from those in non-Japanese patients.

Keywords Lapatinib - Paclitaxel · HER2 - Metastatic breast cancer

7 Department of Breast and Endocrine Surgery, Osaka University Graduate School of Medicine, Osaka, Japan

8 Department of Breast and Endocrine Surgery, Osaka Medical Center for Cancer and Cardiovascular Diseases, Osaka, Japan

9 Department of Breast Oncology, Aichi Cancer Center Hospital, Nagoya, Japan

10 Development and Medical Affairs Division, GlaxoSmithKline K.K., Tokyo, Japan

11 Research and Development, GlaxoSmithKline, Philadelphia, PA, USA

12 Department of Medical Oncology, Saitama Medical University International Medical Center, Saitama, Japan

13 Division of Medical Oncology, Department of Medicine, Showa University Hospital, Tokyo, Japan 


\section{Introduction}

Overexpression or gene amplification of human epidermal growth factor receptor 2 (HER2) occurs in approximately $20 \%$ of breast cancer cases. It is known to be an independent prognostic factor that shortens survival of patients, and is associated with high rates of cell proliferation and lymph node metastases $[1,2]$. At present, the standard regimens used worldwide are the trastuzumab-containing regimens, which have significantly improved the prognosis for HER2positive breast cancer [3,4]. One of the key combination regimens containing trastuzumab is with taxane and is recommended by clinical practice guidelines globally [3-5]. However, the majority of patients experience tumor recurrences and/or metastases and, hence, further treatment options for primary care as well as in the metastatic setting are required. Another important issue is the occurrence of brain metastases due to the inability of trastuzumab to cross the intact blood-brain barrier. Cardiotoxicity has been recognized as one of the important side-effects [6-11]. Lapatinib tosylate hydrate is a small molecule that reversibly inhibits the activity of epidermal growth factor receptor (EGFR) and HER2 tyrosine kinases. Lapatinib was first approved in the USA in 2006 in combination with capecitabine for HER2positive metastatic breast cancer (MBC) which persisted or recurred after anthracycline, taxane and trastuzumab treatment. The approved condition is the same worldwide, including in Japan. Lapatinib has been evaluated in other combinations, such as trastuzumab, aromatase inhibitor and paclitaxel. Its combination with paclitaxel is approved in some countries/regions as first-line treatment for HER2-positive MBC patients in whom trastuzumab is not appropriate.

Lapatinib is a small molecule that passes through the compromised blood-brain barrier and is suggested to exert anti-tumor activity in metastatic brain lesions [12, 13]. This characteristic approach of lapatinib led to the development of lapatinib in combination with paclitaxel as first-line therapy of HER2-positive MBC, as a replacement for the trastuzumab regimens.

The first Phase I study of a lapatinib and paclitaxel combination $(\mathrm{L}+\mathrm{P})$ in HER2-positive MBC patients was conducted outside Japan. This study demonstrated the tolerability of lapatinib $(1500 \mathrm{mg} /$ day $)$ in combination with both weekly paclitaxel $80 \mathrm{mg} / \mathrm{m}^{2}$ and with tri-weekly paclitaxel 135, 175, 200 and $225 \mathrm{mg} / \mathrm{m}^{2}$ [14]. Following this, the combination of lapatinib $(1500 \mathrm{mg} / \mathrm{day})$ and tri-weekly paclitaxel $\left(175 \mathrm{mg} / \mathrm{m}^{2}\right)$ as first-line therapy was evaluated in a Phase III study, in which 579 HER2negative or unknown, advanced or recurrent breast cancer patients were enrolled [15]. Of 579 patients enrolled, 83 had HER2-positive MBC, and the combination regimen showed significant improvement in progression-free survival (PFS) compared to paclitaxel monotherapy [hazard ratio $(\mathrm{HR})=0.49 ; 95 \%$ confidence interval $(\mathrm{CI}) 0.3-0.8$; $p=0.008]$. In another Phase III study that was mainly conducted in China and South America, the combination of lapatinib $(1500 \mathrm{mg} /$ day $)$ with weekly paclitaxel $(80 \mathrm{mg} /$ $\mathrm{m}^{2}$ ) as first-line therapy was evaluated in 444 patients with HER2-positive MBC [16]. The overall survival (OS) of the combination was prolonged compared to paclitaxel monotherapy (HR $=0.74 ; 95 \%$ CI $0.58-0.94 ; p=0.0124)$. The combinations of lapatinib $(1250 \mathrm{mg} /$ day $)$ or trastuzumab either with paclitaxel or docetaxel as first-line therapy for HER2-positive MBC were evaluated in a global Phase III study including Japan, which was conducted mainly in the USA/EU [17]. The study enrolled 636 patients; the results, reported in 2012, showed that the PFS of the lapatinib combination was not significantly different compared with the trastuzumab regimen $[\mathrm{HR}=1.33(95 \%$ CI 1.06-1.67); $p=0.01]$.

In order to extrapolate the data from the above studies conducted outside Japan to clinical practice in Japan, we have conducted this study to investigate the tolerability, safety and pharmacokinetics (PK) of a lapatinib $1500 \mathrm{mg}$ and weekly paclitaxel combination.

\section{Patients and methods}

\section{Patients}

Eligible patients were Japanese women older than 18 years with histologically confirmed invasive HER2-positive MBC, which was defined as immunohistochemistry (IHC) $3+$ or fluorescence in situ hybridization (FISH)-positive (an amplification ratio $\geq 2.2$ ) evaluated by a local laboratory. Patients were required to have at least one measurable lesion according to the Response Evaluation Criteria in Solid Tumors (RECIST) version 1.0 [18]. Patients must have received no prior therapy for metastatic diseases. Additional inclusion criteria were: Eastern Cooperative Oncology Group performance status of 0 or 1 ; left ventricular ejection fraction (LVEF) within the institutional normal range (or $\geq 50 \%$, if unavailable); adequate renal, hepatic and hematologic functions.

Major exclusion criteria were: prior therapy with an EGFR and/or HER2 inhibitor other than trastuzumab; unresolved/unstable, serious toxicity from prior therapy with investigational drug and/or anticancer treatment; uncontrolled infection; $\geq$ Grade 2 peripheral neuropathy according to National Cancer Institute Common Terminology Criteria for Adverse Events (NCI-CTCAE) version 3.0; malabsorption syndrome or other conditions that would prevent the efficacy and safety evaluation of the study regimen. All patients provided written informed consent. The study was conducted in accordance with good clinical 
practice and all applicable regulatory requirements, including the Declaration of Helsinki.

\section{Study design}

This was a single-arm, two-part, open-label Phase I/II study (ClinicalTrials.gov Identifier: NCT01138046). The objectives were to evaluate the tolerability, safety, efficacy and $\mathrm{PK}$ of $\mathrm{L}+\mathrm{P}$ in Japanese patients with HER2-positive MBC who have not received prior chemotherapy or trastuzumab for metastatic diseases. Six patients were enrolled in the first part (Part 1) to evaluate the tolerability of this regimen, and once the tolerability was confirmed, a further 6 patients were enrolled in the second part (Part 2). Sample size was not decided by statistical considerations.

Patients were given the standard treatment consisting of 6 cycles of weekly paclitaxel $80 \mathrm{mg} / \mathrm{m}^{2}$ (for 3 weeks in a 4-week cycle) combined with lapatinib $1500 \mathrm{mg}$ daily; the latter was given until disease progression or withdrawal from study treatment due to unacceptable toxicity or consent withdrawal. The investigators could chose to continue concurrent paclitaxel administration for more cycles. For patients enrolled in Part 1, lapatinib was not given on day 1, but started on the following day, and paclitaxel was not given on day 15 , but was given within 2 days after that date during the first cycle for PK evaluation purposes. If disease progression, unacceptable paclitaxel-related toxicities, or termination of lapatinib occurred, then paclitaxel was terminated at any time during the study, even before completing 6 cycles.

\section{Study endpoints}

The objective in Part 1 was tolerability. If one or no patient out of the 6 experienced any events included in the tolerability criteria, the study treatment was concluded to be tolerable. PK parameters were also evaluated.

The efficacy and safety of the treatment in twelve patients were the objectives of Part 2 . The study was not designed based on statistical hypotheses, as the study targeted a small population and was designed as single-arm stud;, however, in order to compare with the OS of the previous study, our efficacy evaluation was primarily focused on OS. The other endpoints of efficacy were PFS, time to response, duration of response, objective tumor response rate (ORR) and clinical benefit rate [complete response (CR) or partial response (PR) or stable disease (SD) $\geq 24$ weeks]. Safety and biomarkers were also evaluated. All these endpoints were evaluated in the patients enrolled in both parts.

\section{Safety and efficacy assessments}

Safety assessment including laboratory tests were performed every week during the combination treatment and at the discontinuation of paclitaxel if it was decided to continue longer than 6 cycles. If paclitaxel was discontinued at the sixth cycle, then safety assessment was conducted every 8 weeks until the end of treatment. LVEF assessment by echocardiogram was performed at the end of evennumbered cycles during combination treatment and every 8 weeks while on lapatinib monotherapy. Adverse events (AEs) were graded according to NCI-CTCAE version 3.0. AE terms were coded by MedDRA Ver13.1. The protocol defined serious AEs as all Grade 4 laboratory abnormalities, Grade 3 or 4 decrease in LVEF, $\geq 20 \%$ decrease in LVEF relative to baseline and also below the institution's lower limit of normal (if the lower limit of normal was unavailable, decrease to less than $50 \%$ ), Grade 3 pneumonitis, alanine aminotransferase (ALT) $>3 \times$ upper limit of normal (ULN), and total bilirubin $>2.0 \times \mathrm{ULN}$ ( $>35 \%$ direct; bilirubin fractionation required). The tolerability criteria were defined as the toxicities related to study treatment and applicable to any of the following: Grade 4 neutropenia sustained for $\geq 7$ days, Grade 4 thrombocytopenia, $\geq$ Grade 3 or clinically significant non-hematologic toxicities (other than nausea), or unable to start cycle 2 within 2 weeks of scheduled dosing due to unresolved toxicity. Patients withdrawn from the study without disease progression were assessed every 12 weeks until progression, start of postanticancer therapy or death. Efficacy assessment was performed at baseline and at the ends of every even-numbered cycle until withdrawal from the study. Tumor response was assessed by the investigators, using images or photographic data, in accordance with the RECIST [18]. Biomarker analysis for HER2 status was conducted based on the results determined by both IHC and FISH at the central laboratory.

\section{Pharmacokinetics evaluation}

Pharmacokinetics of lapatinib and/or paclitaxel were evaluated in all patients enrolled in Part 1 on days 1, 8 and 14: day 14 for PK of lapatinib monotherapy, day 1 for paclitaxel monotherapy and day 8 for combination therapy. Plasma samples were taken pre-dose and at $0.5,1,1.5,2$, $3,4,6,8,12$ and $24 \mathrm{~h}$ post-dose. The PK parameters calculated by non-compartmental analysis were maximum plasma concentration $\left(C_{\max }\right)$, time to $C_{\max }\left(t_{\max }\right)$ and area under the plasma concentration curve (AUC) from 0 to $24 \mathrm{~h}\left(\mathrm{AUC}_{0-24}\right)$ of lapatinib as well as $C_{\text {max }}, t_{\max }$, half-life, AUC extrapolated to infinity $\left(\mathrm{AUC}_{0-\mathrm{inf}}\right)$ and $\mathrm{AUC}_{0-24}$ of paclitaxel.

\section{Statistical analysis}

The sample size was determined based on study feasibility. This study did not assert or test any statistical hypotheses. 
The dose-limiting toxicity (DLT) incidences were evaluated in 6 patients. The intent-to-treat (ITT) population was analyzed for safety and efficacy analyses. PFS and OS were summarized using the Kaplan-Meier method. All the patients who had provided ample plasma samples for the PK parameter evaluation were treated as the PK population. For PK parameters, the items evaluated are shown in Table 5 .

Version 9.1.3 Unix SAS $^{\circledR}$ system (a registered trademark of the SAS Institute, Inc., Cary, NC, USA) was used for analysis.

\section{Results}

\section{Patient characteristics}

A total of 12 patients were enrolled from 9 centers between April 2010 and June 2011, and were treated with the study regimen. As of 31 January 2014 (the final data cut-off date), 6 patients had completed the study and 6 patients were followed up for survival.

Out of 12 patients enrolled, 8 patients had both visceral and non-visceral metastatic lesions, 2 patients had visceral lesions only, while the other 2 patients had nonvisceral lesions only (Table 1). The median time since diagnosis was 12.9 months; 4 patients had received prior chemotherapy, of whom one had received prior trastuzumab. Six patients had estrogen receptor (ER)-positive breast cancer as assessed by a local laboratory, of whom 4 patients were positive for both ER and progesterone receptor.

\section{Tolerability and safety}

The median duration of lapatinib treatment was 50.9 weeks (range 4-117 weeks). Toxicities other than hematologic or neurologic toxicities leading to dose reduction occurred in 4 patients; however, DLT was not observed. The numbers of dose reductions observed were once $(1250 \mathrm{mg})$ in 2 patients, twice $(1000 \mathrm{mg})$ in 1 patient and three times (750 $\mathrm{mg}$ ) in 1 patient. The primary reasons for dose reduction were rash, acne, diarrhea, increased ALT and increased aspartate aminotransferase (AST). Dose interruptions of lapatinib were reported in 10 patients, 73 times in total, mainly due to hematologic or neurologic toxicities. The median duration of interruption was 7 days (range 1-21 days).

For paclitaxel, the median number of cycles was 10 cycles (range 2-36 cycles), in which eight patients received more than 6 cycles. Neurologic toxicity was the cause of the dose reduction in one patient and of the dose interruptions of paclitaxel in 10 patients. All 12
Table 1 Baseline characteristics of intent-to-treat population

\begin{tabular}{ll}
\hline $\begin{array}{l}\text { Age, years } \\
\text { Median (range) }\end{array}$ & $59.0(45-70)$ \\
Time since diagnosis (months) & \\
Median (min-max) & $12.9(0-115)$ \\
1st Quartile & 1.2 \\
3rd Quartile & 76.4 \\
Prior anti-cancer therapy, $n(\%)$ & \\
Chemotherapy & $4(33)$ \\
Anthracyclines & $1(8)$ \\
$\quad$ Taxanes & $3(25)$ \\
$\quad$ Trastuzumab & $1(8)$ \\
Surgery & $6(50)$ \\
Radiotherapy & $2(17)$ \\
Endocrine therapy & $4(33)$ \\
Immunotherapy & 0 \\
Metastatic sites, $n(\%)$ & \\
Visceral & $2(17)$ \\
Non-visceral & $2(17)$ \\
Visceral and non-visceral & $8(67)$ \\
Hormone receptor status, $n(\%)$ & \\
ER+ and/or PgR+ & \\
ER+ and PgR+ & $6(50)$ \\
ER+ and PgR- & $4(33)$ \\
ER- and PgR- & $2(17)$ \\
Unknown & $6(50)$ \\
\hline
\end{tabular}

Based on diagnosis made by investigators

$E R$ estrogen receptor, $P g R$ progesterone receptor

patients were withdrawn from $\mathrm{L}+\mathrm{P}$, mostly due to disease progression.

In Part 1 , the tolerability and safety of the study treatment in Japanese patients were confirmed. All patients experienced at least one AE regardless of the relationship with the study treatments, and most of them were at Grades 1 or 2 . The most common AEs reported were alopecia, neutropenia, diarrhea, decreased hemoglobin and rash (Table 2). Grade 3 treatment-related AEs found in more than 2 patients were: neutropenia $(n=7)$, leukopenia $(n=5)$, diarrhea $(n=3)$, increased ALT $(n=3)$ and increased AST $(n=2)$. A Grade 4 treatment-related event, neutropenia, occurred in 2 patients.

Rash and diarrhea were the special interest AEs for lapatinib. No $\geq$ Grade 3 or serious rash was reported. One Grade 2 rash event led to withdrawal from study treatment in one patient who had concurrently experienced Grade 2 erythema of the eyelid and on the back of both hands. Although Grade 3 diarrhea events occurred in 3 patients, no diarrhea was reported as $\geq$ Grade 4 or serious, and there was no withdrawal from study treatment due to diarrhea. 
Table 2 Summary of adverse events with at least $50 \%$ occurrence

\begin{tabular}{lllccl}
\hline Adverse event, $n(\%)$ & Grade 1 & Grade 2 & Grade 3 & Grade 4 & Total \\
\hline Alopecia & $6(50)$ & $6(50)$ & 0 & 0 & $12(100)$ \\
Diarrhea & $4(33)$ & $4(33)$ & $3(25)$ & 0 & $11(92)$ \\
Neutropenia & 0 & $2(17)$ & $7(58)$ & $2(17)$ & $11(92)$ \\
$\begin{array}{l}\text { Decreased } \\
\text { hemoglobin }\end{array}$ & $3(25)$ & $6(50)$ & $1(8)$ & 0 & $10(83)$ \\
Rash & $6(50)$ & $3(25)$ & 0 & 0 & $9(75)$ \\
Stomatitis & $8(67)$ & 0 & 0 & 0 & $8(67)$ \\
Fatigue & $8(67)$ & 0 & 0 & 0 & $8(67)$ \\
Peripheral sensory & $6(50)$ & $2(17)$ & 0 & 0 & $8(67)$ \\
$\quad$ neuropathy & & & & & \\
Leukopenia & 0 & $3(25)$ & $5(42)$ & 0 & $8(67)$ \\
Decreased apatite & $4(33)$ & $3(25)$ & 0 & 0 & $7(58)$ \\
Paronychia & $5(42)$ & $2(17)$ & 0 & 0 & $7(58)$ \\
ALT Increased & 0 & $4(33)$ & $3(25)$ & 0 & $7(58)$ \\
AST Increased & $1(8)$ & $3(25)$ & $2(17)$ & 0 & $6(50)$ \\
Lymphopenia & $1(8)$ & $4(33)$ & $1(8)$ & 0 & $6(50)$ \\
Decreased & $5(42)$ & $1(8)$ & 0 & 0 & $6(50)$ \\
$\quad$ hematocrit & & & & & \\
Vomiting & $4(33)$ & $2(17)$ & 0 & 0 & $6(50)$ \\
Nasopharyngitis & $5(42)$ & $1(8)$ & 0 & 0 & $6(50)$ \\
Nail disorder & $4(33)$ & $2(17)$ & 0 & 0 & $6(50)$ \\
\hline
\end{tabular}

$A L T$ alanine aminotransferase, $A S T$ aspartate aminotransferase

No fatal serious AE was reported. Four protocoldefined serious AEs were reported in 3 patients; these were decreased neutrophil count in 2 patients, left ventricular dysfunction in a patient with a history of prior anthracycline treatment for other past malignancy, and pneumonia in a patient who was diagnosed by X-ray imaging. All these were considered by investigators to be treatment-related. Although the follow-up of left ventricular dysfunction was discontinued due to the start of another treatment, other serious AEs resolved within 2 weeks.

\section{Efficacy}

As of the end of the study, 6 patients had died. The remaining 6 patients were censored at the last visit. The median OS as primary endpoint was 35.6 months (95\% CI 23.9, not reached; Fig. 1). PFS was analyzed using the results evaluated by the investigators and the median was 13.9 months (95 \% CI 7.6, 27.9; Fig. 2).

Ten out of 12 patients $(83 \%)$ achieved clinical benefit (95\% CI 51.6, 97.9) based on the investigators' assessment (Table 3). The ORR in the ITT population was $83 \%(95 \%$ CI 51.6, 97.9) with $10 \mathrm{PRs}$, while one patient had SD for less than 24 weeks and progressive disease was observed in one patient.

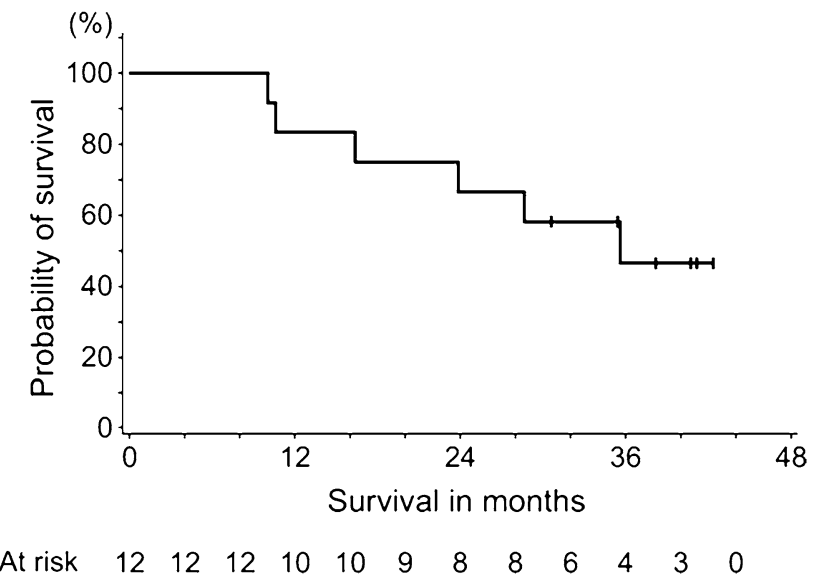

Fig. 1 Kaplan-Meier estimates for overall survival

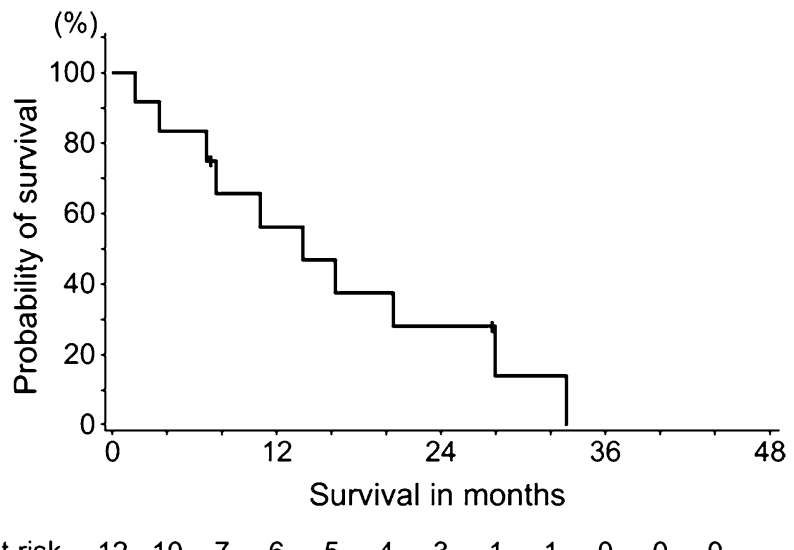

$\begin{array}{lllllllllllll}\text { At risk } & 12 & 10 & 7 & 6 & 5 & 4 & 3 & 1 & 1 & 0 & 0 & 0\end{array}$

Fig. 2 Kaplan-Meier estimates for progression-free survival assessed by investigators

\section{Pharmacokinetics}

The plasma concentration-time profile of lapatinib after repeated oral dosing of lapatinib $1500 \mathrm{mg}$ with or without concomitant administration of paclitaxel is shown in Fig. 3, and the plasma concentration-time profile of paclitaxel after $1 \mathrm{~h}$ intravenous infusion of paclitaxel $80 \mathrm{mg} / \mathrm{m}^{2}$ with or without concomitant administration of lapatinib is shown in Fig. 4.

The geometric means of $C_{\max }$ and $\mathrm{AUC}_{(0-24)}$ of lapatinib were increased by 59 and $42 \%$ under the paclitaxel $80 \mathrm{mg} / \mathrm{m}^{2}$ combination, in comparison to lapatinib alone. No change was observed in $t_{\max }$ (Table 4). The geometric means of $C_{\max }$ and $t_{\max }$ of paclitaxel were not changed by combination with lapatinib; however, that of $\mathrm{AUC}_{(0-\text { inf })}$ was increased by $23 \%$ (Table 5). 
Table 3 Summary of tumor response in intent-to-treat population

\begin{tabular}{ll}
\hline Best response, $n(\%)$ & \\
\hline CR & 0 \\
PR & $10(83)$ \\
SD, $\geq 24$ weeks & 0 \\
SD, <24 weeks & $1(8)$ \\
PD & $1(8)$ \\
NE & 0 \\
ORR & $83(95 \%$ CI 51.6, 97.9) \\
CBR & $83(95 \%$ CI 51.6, 97.9)
\end{tabular}

$C B R$ clinical benefit rate (CR; $\mathrm{PR}$; $\mathrm{SD} \geq 24$ weeks), $C R$ complete response, $N E$ not evaluable, $O R R$ overall tumor response rate, $P D$ progressive disease, $P R$ partial response, $S D$ stable disease

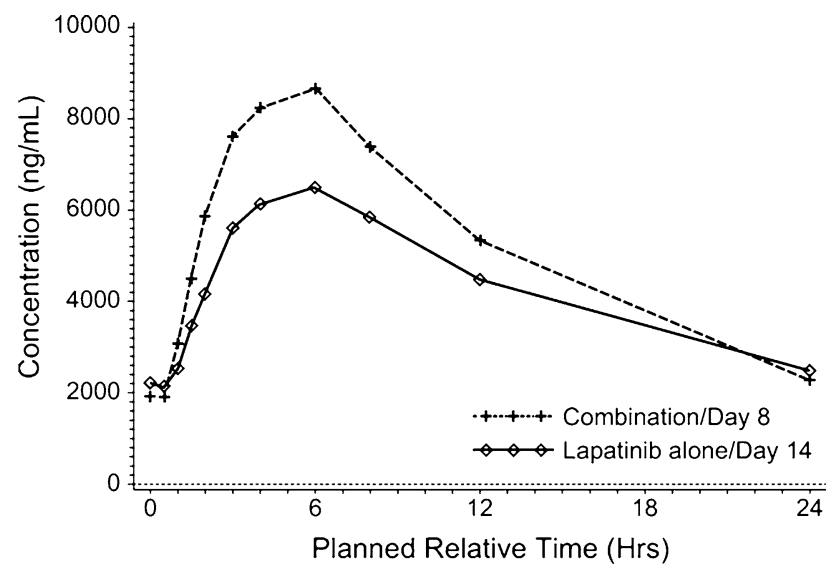

Fig. 3 Plasma concentration-time profile of lapatinib after dosing of lapatinib $1500 \mathrm{mg}$ with or without concomitant administration of paclitaxel $80 \mathrm{mg} / \mathrm{m}^{2}$

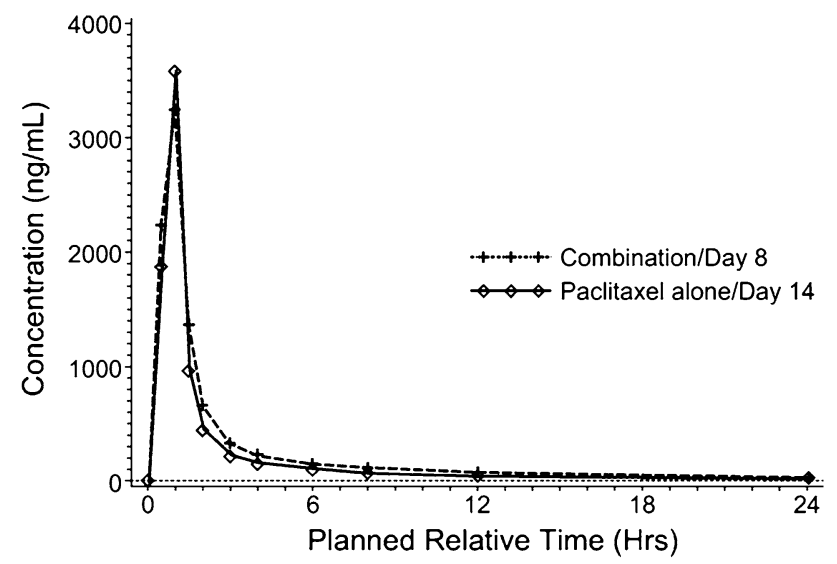

Fig. 4 Plasma concentration-time profile of paclitaxel after dosing of paclitaxel $80 \mathrm{mg} / \mathrm{m}^{2}$ with or without concomitant administration of lapatinib $1500 \mathrm{mg}$

\section{Discussion}

The tolerability of the lapatinib (1500 mg/day) and weekly paclitaxel $\left(80 \mathrm{mg} / \mathrm{m}^{2}\right)$ combination was confirmed in Japanese patients with HER2-positive MBC. Reported results from the pilot part of the Asian Phase III study targeting gastric cancer patients showed a similar tolerability of this regimen [19].

Drug-drug interaction of lapatinib and paclitaxel was found, as the AUC and $C_{\max }$ of Japanese breast cancer patients given the combination were affected. A similar trend of interaction was observed in a Phase I study targeting solid tumor patients conducted outside of Japan [14]. The extent of drug interaction of $\mathrm{L}+\mathrm{P}$ was also similar to those of the pilot part of the Asian Phase III study which included patients with a history of gastrectomy [19]. The interaction found in PK profiles of lapatinib and paclitaxel is consistent regardless of the cancer type, and such interaction was considered to be the result of lapatinib being a weak metabolism-dependent inhibitor of CYP3A4.

The AEs reported in this study were generally consistent with those reported in overseas clinical studies evaluating $\mathrm{L}+\mathrm{P}$ and with known safety profiles of lapatinib and paclitaxel given as monotherapy [14-17]. The grade of diarrhea was slightly worsened compared with lapatinib monotherapy, which means that $\mathrm{L}+\mathrm{P}$ would require more careful management in clinical practice.

Our study demonstrated that the combination therapy of $\mathrm{L}+\mathrm{P}$ was efficacious for the treatment of HER2-positive $\mathrm{MBC}$, which was consistent with the results of a Phase III study [16]. Meanwhile, results became available from another global Phase III study which showed a significant PFS of a trastuzumab-containing regimen compared with a lapatinib and taxane regimen [17]. Moreover, the results of a trastuzumab, docetaxel and pertuzumab tri-regimen became available after the results of that global study were reported, and, up to 2014, this tri-regimen became the standard in first-line therapy of MBC worldwide [20]. As the new treatment of trastuzumab, pertuzumab and docetaxel tri-regimen became available for $\mathrm{MBC}$ and the results of direct comparison between lapatinib and trastuzumab were confirmed, it is now proven to be difficult to recommend $\mathrm{L}+\mathrm{P}$ as the first-line therapy, which we originally expected.

Overall, our study provides valuable results that show the drug-drug interaction and $\mathrm{PK}$ interaction between lapatinib and paclitaxel in Japanese patients with MBC. Although our study does not impact upon the clinical positions or the treatment strategy, it confirms that the combination of lapatinib with paclitaxel is tolerable in Japanese 
Table 4 Pharmacokinetic parameters of lapatinib after repeat dosing with or without concomitant administration of paclitaxel

\begin{tabular}{|c|c|c|c|}
\hline Parameter & Lapatinib $1500 \mathrm{mg}$ alone $(n=6)$ & Lapatinib $1500 \mathrm{mg}+$ paclitaxel $80 \mathrm{mg} / \mathrm{m}^{2}(n=6)$ & Ratio $(90 \% \mathrm{CI})^{\mathrm{a}}$ \\
\hline$C_{\max }(\mathrm{ng} / \mathrm{mL})$ & $5945.0(3077.9,11483.0)$ & $9470.4(7157.9,12530.0)$ & $1.59(0.96,2.64)$ \\
\hline$t_{\max }(\mathrm{h})$ & $4.992^{\mathrm{b}}(1.50,6.08)^{\mathrm{c}}$ & $4.975^{\mathrm{b}}(1.95,6.00)^{\mathrm{c}}$ & $0.00^{\mathrm{d}}(-2.06,2.21)$ \\
\hline $\operatorname{AUC}_{(0-24)}(\mathrm{ng} \cdot \mathrm{h} / \mathrm{mL})$ & $79518.0(34664.6,182408.5)$ & $113078.3(74630.1,171334.3)$ & $1.42(0.82,2.46)$ \\
\hline
\end{tabular}

Geometric mean $(95 \% \mathrm{CI})$

$A U C_{(0-24)}$ area under the curve from 0 to $24 \mathrm{~h}, C I$ confidence interval, $C_{\max }$ maximum plasma concentration, $t_{\max }$ time to reach maximum concentration

${ }^{\mathrm{a}}$ Ratio $=($ lapatinib + paclitaxel $) /$ lapatinib alone

${ }^{\mathrm{b}}$ Median

${ }^{c}$ Min and max

${ }^{\mathrm{d}}$ Median difference

Table 5 Pharmacokinetic parameters of paclitaxel after dosing with or without concomitant administration of lapatinib

\begin{tabular}{llll}
\hline Parameter & Paclitaxel $80 \mathrm{mg} / \mathrm{m}^{2}$ alone $(n=6)$ & Paclitaxel $80 \mathrm{mg} / \mathrm{m}^{2}+$ lapatinib $1500 \mathrm{mg}(n=6)$ & Ratio $(90 \%$ CI $)$ \\
\hline$C_{\max }(\mathrm{ng} / \mathrm{mL})$ & $3485.2(2693.0,4510.5)$ & $3412.3(2753.1,4229.4)$ & $0.98(0.85,1.13)$ \\
$t_{\max }(\mathrm{h})$ & $0.992^{\mathrm{b}}(0.95,1.08)^{\mathrm{c}}$ & $0.975^{\mathrm{b}}(0.50,1.02)^{\mathrm{c}}$ & $-0.05^{\mathrm{d}}(-0.27,0.00)$ \\
$\mathrm{AUC}_{(0-24)}(\mathrm{ng} . \mathrm{h} / \mathrm{mL})$ & $4657.2(3942.6,5501.3)$ & $5786.1(4667.5,7172.8)$ & $1.24(1.13,1.36)$ \\
$\mathrm{AUC}_{(0-\text { inf })}(\mathrm{ng} . \mathrm{h} / \mathrm{mL})$ & $5125.9(4371.3,6010.8)$ & $6280.0(5005.6,7878.8)$ & $1.23(1.10,1.37)$ \\
$t_{1 / 2}(\mathrm{~h})$ & $12.19(10.06,14.77)$ & & $9.86(8.48,11.46)$ \\
& & & $0.81(0.71,0.92)$ \\
\hline
\end{tabular}

Geometric mean $(95 \% \mathrm{CI})$

$A U C_{(0-24)}$ area under the curve, $A U C_{(0-i n f)}$ area under the curve extrapolated to infinity, $C_{\max }$ maximum plasma concentration, $C I$ confidence interval, $t_{\max }$ time to reach maximum plasma concentration, $t_{1 / 2}$ half-life

${ }^{\mathrm{a}}$ Ratio $=($ paclitaxel + lapatinib $) /$ paclitaxel alone

${ }^{\mathrm{b}}$ Median

${ }^{c}$ Min and max

${ }^{\mathrm{d}}$ Median difference

patients with MBC. Nevertheless, paclitaxel remains a key drug in breast cancer therapies and many multipleagent regimens with paclitaxel have been evaluated worldwide. For this, it is considered that our findings of PK and safety data of L+P may be beneficial to those who seek the appropriate dose and safety management of the regimen. Currently, studies targeting the adjuvant/neoadjuvant setting in which to evaluate combinations such as lapatinib, paclitaxel and trastuzumab are ongoing. There is no evidence to recommend the use of lapatinib in comparison to trastuzumab; however, our data can be utilized for considering the best practice of HER2 targeting therapies.

In conclusion, $\mathrm{L}+\mathrm{P}$ was tolerable in Japanese patients with MBC, with manageable safety profiles, and a similar trend of the interaction of $\mathrm{L}+\mathrm{P}$ to that previously reported in other ethnicities, as well as in different cancer types, was found.

Acknowledgments We thank all the patients who participated in this study and their families; the investigators; medical nurses and research staffs at all the study centers. This study was fully funded and lapatinib was supplied by GlaxoSmithKline K.K.

Conflict of interest Kenichi Inoue, Katsumasa Kuroi, Satoru Shimizu, Yoshiaki Rai, Kenjiro Aogi, Norikazu Masuda, Takahiro Nakayama, Hiroji Iwata, Yasutsuna Sasaki had no conflicts of interest; Alison Armour and Yuichiro Nishimura are employees of GlaxoSmithKline.

Open Access This article is distributed under the terms of the Creative Commons Attribution 4.0 International License (http://creativecommons.org/licenses/by/4.0/), which permits unrestricted use, distribution, and reproduction in any medium, provided you give appropriate credit to the original author(s) and the source, provide a link to the Creative Commons license, and indicate if changes were made.

\section{References}

1. Ross JS, Slodkowska EA, Symmans WF et al (2009) The HER-2 receptor and breast cancer: ten years of targeted anti-HER-2 therapy and personalized medicine. Oncologist 14:320-368 
2. Burstein HJ, Winer EP (2005) The distinctive nature of HER2 positive breast cancer. N Engl J Med 353:1652-1654

3. Giordano SH, Temin S, Kirshner JJ et al (2014) Systemic therapy for patients with advanced human epidermal growth factor receptor 2-positive breast cancer: American Society of Clinical Oncology clinical practice guideline. J Clin Oncol 32:2078-2099

4. National comprehensive cancer network (2014) Clinical practice guidelines in oncology. Breast cancer. Version 3

5. Japanese Breast Cancer Society (2013) Evidence based breast cancer diagnostic guideline. Japanese Breast Cancer Society, editors. 1 Medicinal Therapy. 2013 version. Tokyo: Kanehara Shuppan, Tokyo

6. Bendell JC, Domchek SM, Burstein HJ et al (2003) Central nervous system metastases in women who receive trastuzumab-based therapy for metastatic breast carcinoma. Cancer 97:2972-2977

7. Clayton AJ, Danson S, Jolly S et al (2004) Incidence of cerebral metastases in patients treated with trastuzumab for metastatic breast cancer. Br J Cancer 91:639-643

8. Lin NU, Winer EP (2007) Brain metastases: the HER2 paradigm. Clin Cancer Res 13:1648-1655

9. Stemmler HJ, Kahlert S, Siekiera W et al (2006) Characteristics of patients with brain metastases receiving trastuzumab for HER2 overexpressing metastatic breast cancer. Breast $15: 219-225$

10. Yau T, Swanton C, Chua $S$ et al (2006) Incidence, pattern and timing of brain metastases among patients with advanced breast cancer treated with trastuzumab. Acta Oncol 45:196-201

11. Nielsen DL, Kumler I, Palshof AE et al (2013) Efficacy of HER2-targeted therapy in metastatic breast cancer. Monoclonal antibodies and tyrosine kinase inhibitors. Breast 22:1-12

12. Geyer C, Forster J, Lindquist D et al (2006) Lapatinib plus capecitabine for HER2-positive advanced breast cancer. N Engl J Med 355:2733-2743
13. Lin NU, Carey LA, Liu MC et al (2008) Phase II trial of lapatinib for brain metastases in patients with human epidermal growth factor receptor 2-positive breast cancer. J Clin Oncol 26:1993-1999

14. Jones SF, Hainsworth JD, Spigel DR, et al (2004) A phase I study of the dual kinase inhibitor GW572016 in combination with paclitaxel (EGF10009). J Clin Oncol 22(14S): (Abstract 2083)

15. Di Leo A, Gomez HL, Aziz Z et al (2008) Phase III, doubleblind, randomized study comparing lapatinib plus paclitaxel with placebo plus paclitaxel as first-line treatment for metastatic breast cancer. J Clin Oncol 26:5544-5552

16. Guan Z, Xu B, DeSilvio ML et al (2013) Randomized trial of lapatinib versus placebo added to paclitaxel in the treatment of human epidermal growth factor receptor 2-overexpressing metastatic breast cancer. J Clin Oncol 31:1947-1953

17. Gelmon KA, Boyle F, Kaufman B, et al (2012) Open-label phase III randomized controlled trial comparing taxane-based chemotherapy (Tax) with lapatinib (L) or trastuzumab (T) as first-line therapy for women with HER2 + metastatic breast cancer: Interim analysis (IA) of NCIC CTG MA.31/GSK EGF 108919. J Clin Oncol 30 (suppl):LBA671

18. Therasse P, Arbuck SG, Eisenhauer EA et al (2000) New guidelines to evaluate the response to treatment in solid tumors. J Natl Cancer Inst 92:205-216

19. Satoh T, Xu RH, Chung HC et al (2014) Lapatinib plus paclitaxel versus paclitaxel alone in the second-line treatment of HER2amplified advanced gastric cancer in Asian populations: TyTANa randomized, phase III study. J Clin Oncol 32(19):2039-2049

20. Baselga J, Cortes J, Kim SB et al (2012) Pertuzumab plus trastuzumab plus docetaxel for metastatic breast cancer. N Engl J Med 366:109-119 